\title{
Global Attractor and Dimension Estimation for a 2D Generalized Anisotropy Kuramoto-Sivashinsky Equation
}

\author{
Meixia Wang, Cuicui Tian, Guoguang Lin \\ Department of Mathematics, Yunnan University, Kunming, China \\ Email: zhuzhicao@163.com, 880903tc@163.com, gglin@ynu.edu.cn
}

Received 5 August 2014; revised 2 September 2014; accepted 8 September 2014

Copyright (C) 2014 by authors and Scientific Research Publishing Inc.

This work is licensed under the Creative Commons Attribution International License (CC BY). http://creativecommons.org/licenses/by/4.0/

(c) () Open Access

\section{Abstract}

In this paper, firstly, some priori estimates are obtained for the existence and uniqueness of solutions of a two dimensional generalized anisotropy Kuramoto-Sivashinsky Equation. Then we prove the existence of the global attractor. Finally, we get the upper bound estimation of the Haus-dorff and fractal dimension of attractor.

\section{Keywords}

\section{Kuramoto-Sivashinsky Equation, Existence, Global Attractor, Dimension Estimation}

\section{Introduction}

In recent years, the infinite dimension dynamic system with high dimension has been studied extensively, and the studies have obtained many achievements [1]-[8]. The related questions of its existence and uniqueness of solutions; the existence and dimension of global attractor; the existence and attraction of inertial manifolds; finite dimension, approximate inertial manifolds and time-lag inertial manifolds are still important contents that are studied.

The celebrated Kuramoto-Sivashinsky Equation

$$
u_{t}+u_{x x x x}+u_{x x}+\frac{1}{2} u_{x}^{2}=0
$$

where $u=u(x, t)$, is an Equation that for nearly half a century has attracted the attention of many researchers from various areas due to its simple but rich dynamics [9]. It first appeared in the mid-1970s by Kuramoto in the study of angularphase turbulence for a system of reaction-diffusion equations modeling the Belousov Zhabo- 
tinskii reaction in three spatial dimensions [10].

In a physical context, Equation (1.1) is used to model continuous media that exhibits chaotic behavior such as weak turbulence on interfaces among complex flows (quasi-planar flame front and the fluctuation of the positions of a flame front, fluctuations in thin viscous fluid films flowing over inclined planes or vertical walls, dendritic phase change fronts in binary alloy mixtures), small perturbations of a metastable planar front or interface (spatially uniform oscillating chemical reaction in a homogeneous medium) and physical systems driven far from the equilibrium due to intrinsic instabilities (instabilities of dissipative trapped ion modes in plasmas and phase dynamics in reaction-diffusion systems).

As a dynamical system the KSE is known for its chaotic solutions and complicated behavior due to the terms that appear. Namely, the $u_{x x}$ term acts as an energy source and has a destabilizing effect at a large scale, the dissipative $u_{x x x x}$ term provides dumping in small scales and, finally, the nonlinear term provides stabilization by transferring energy between large and small scales. Because of this fact, Equation (1.1) was studied extensively as a paradigm of finite dynamics in a partial differential equation. Its multi-modal, oscillatory and chaotic solutions have been investigated; its non-integrability was established via its Painlev analysis and due to its bifurcation behavior, a connection to low finite-dimensional dynamical systems is established.

The generalization of KSE to two dimensions comes naturally, the two-dimensional KuramotoCSivashinsky Equation

$$
u_{t}+\nabla^{4} u+\nabla^{2} u+(\nabla u) \cdot(\nabla u)=0,
$$

where now $u=u(x, y, t)$ and $\nabla^{2}=\nabla \cdot \nabla, \nabla^{4}=\nabla \cdot \nabla(\nabla \cdot \nabla)$. Equation (1.2) has equally attracted much attention because of the same spatiotemporal chaos properties that exhibits and its applications in modeling complex dynamics in hydrodynamics [11]. Nevertheless, due to the additional spatial dimension Equation (1.2) is very challenging and even its well-posedness is still an open problem.

One generalization of Equation (1.2) which is of much interest is the anisotropic two-dimensional KuramotoCSivashinsky Equation

$$
u_{t}=\frac{1}{2} u_{x^{2}}+\frac{\beta}{2} u_{y^{2}}-u_{x x}-\alpha u_{y y}-u_{x x x x}-2 u_{x x y y}-u_{y y y y}
$$

where the two real parameters $\alpha, \beta$ control the anisotropy of the linear and the nonlinear term, respectively, in other words, the stability of the solutions of Equation (1.3). The anisotropic two-dimensional KuramotoCSivashinsky Equation, due to the fact that it describes linearly unstable surface dynamics in the presence of in-plane anisotropy, has a wide range of applications, for instance, as a model for the nonlinear evolution of sputter-eroded surfaces and describing the epitaxial growth of a vicinal surface destabilized by step edge barriers; for further details, see the references therein, in particular [12].

This paper focuses on the following generalization of the anisotropic KSE (1.3)

$$
u_{t}=\frac{1}{2} u_{x^{2}}+h(u) u_{y^{2}}+r(u) u_{x x}+g(u) u_{y y}-u_{x x x x}-2 u_{x x y y}-u_{y y y y}+f(u),
$$

where $f, g, h$ and $r$ are considered as smooth functions of $u=u(x, y, t)$, and its study under the prism of Lie point symmetries and conservation laws [13].

According to the above information, the paper mainly thinks about the following generalization of the anisotropic KSE (1.4)

$$
\begin{aligned}
& u_{t}+\alpha \Delta^{2} u+\gamma u+(\varphi(u))_{x x}+(g(u))_{y y}=f,(x, y) \in \Omega \subset R^{2}, \\
& \left.u(x, y, t)\right|_{t=0}=u_{0}(x, y),(x, y) \in \Omega \subset R^{2}, \\
& \left.u(x, y, t)\right|_{\partial \Omega=0}=0,\left.\Delta u(x, y, t)\right|_{\partial \Omega}=0,(x, y) \in \Omega \subset R^{2} .
\end{aligned}
$$

Here $\Omega \subset R^{2}$ is bounded set; $\partial \Omega$ is the bound of $\Omega ; \varphi(u)$ and $g(u)$ are considered as smooth functions of $u(x, y, t)$. Let $\|\cdot\|=\|\cdot\|_{L^{2}(\Omega)},\|\cdot\|_{\infty}=\|\cdot\|_{L^{\infty}(\Omega)}$.

The following is the rest of this paper. In Section 2, we introduce some basic contents concerning global at- 
tractor. In Section 3, we obtain the existence of the global attractor, then we get the upper bound estimation of the Hausdorff and fractal dimension of the global attractor.

\section{The Priori Estimate of Solution of Questions (1.5) - (1.7)}

Lemma 1. Assume $\left|\varphi^{\prime}(u)\right| \leq \varphi_{0} ; \quad\left|g^{\prime}(u)\right| \leq g_{0}, \quad g_{0} \leq 2 \gamma-\varphi_{0}-1 ; \quad \alpha>\frac{\varphi_{0}+g_{0}}{2} ; \quad f \in L^{2}(\Omega), \quad u_{0} \in L^{2}(\Omega)$, so the smooth solution $u$ of Questions (1.5) - (1.7) satisfies

$$
\|u\|^{2} \leq \mathrm{e}^{\left(\varphi_{0}+g_{0}-2 \gamma+1\right) t}\left\|u_{0}\right\|^{2}+\frac{\|f\|^{2}}{\left|\varphi_{0}+g_{0}-2 \gamma+1\right|} .
$$

Proof. We multiply $u$ with both sides of Equation (1.5) and obtain

$$
\left(u, u_{t}+\alpha \Delta^{2} u+\gamma u+(\varphi(u))_{x x}+(g(u))_{y y}\right)=(u, f),
$$

Here

$$
\begin{gathered}
\left(u, u_{t}\right)=\frac{1}{2} \frac{\mathrm{d}}{\mathrm{d} t}\|u\|^{2},\left(u, \alpha \Delta^{2} u\right)=\alpha\|\Delta u\|^{2},(u, \gamma u)=\gamma\|u\|^{2}, \\
\left(u,(\varphi(u))_{x x}\right)=-\left(u_{x},(\varphi(u))_{x}\right)=-\left(u_{x}, \varphi^{\prime}(u) u_{x}\right)=-\int_{\Omega} \varphi^{\prime}(u) u_{x}^{2} \mathrm{~d} \Omega \geq-\varphi_{0}\|\nabla u\|^{2}, \\
\left(u,(g(u))_{y y}\right)=-\left(u_{y},(g(u))_{y}\right)=-\left(u_{y}, g^{\prime}(u) u_{y}\right)=-\int_{\Omega} g^{\prime}(u) u_{y}^{2} \mathrm{~d} \Omega \geq-g_{0}\|\nabla u\|^{2} .
\end{gathered}
$$

According to Nirenberg-Gagliardo and Cauchy inequality, we obtain

$$
\|\nabla u\|^{2} \leq\|\Delta u\|\|u\| \leq \frac{1}{2}\left(\|\Delta u\|^{2}+\|u\|^{2}\right), \quad(u, f) \leq \frac{1}{2}\left(\|u\|^{2}+\|f\|^{2}\right),
$$

From the (2.2) we obtain

$$
\frac{1}{2} \frac{\mathrm{d}}{\mathrm{d} t}\|u\|^{2}+\left(\alpha-\frac{\varphi_{0}+g_{0}}{2}\right)\|\Delta u\|^{2} \leq\left(\frac{\varphi_{0}+g_{0}+1}{2}-\gamma\right)\|u\|^{2}+\frac{1}{2}\|f\|^{2} .
$$

Using the Gronwall inequality, the (2.1) is proved.

Lemma 2. Under the condition of Lemma 1, and $\left|\varphi^{\prime}(u)\right| \leq A|u|^{q}, 0 \leq q<2 ; \quad\left|g^{\prime}(u)\right| \leq B|u|^{p}, 0 \leq p<2$; $f \in L^{-2}(\Omega), u_{0} \in H^{1}(\Omega)$, so the smooth solution $u$ of Questions (1.5) - (1.7) satisfies

$$
\|\nabla u\|^{2} \leq \mathrm{e}^{-2 \gamma t}\left\|\nabla u_{0}\right\|^{2}+\frac{C_{5}}{\gamma} .
$$

Proof. We multiply $\Delta u$ with both sides of Equation (1.5) and obtain

$$
\left(\Delta u, u_{t}+\alpha \Delta^{2} u+\gamma u+(\varphi(u))_{x x}+(g(u))_{y y}\right)=(\Delta u, f) .
$$

Here

$$
\begin{gathered}
\left(\Delta u, u_{t}\right)=-\frac{1}{2} \frac{\mathrm{d}}{\mathrm{d} t}\|\nabla u\|^{2},\left(\Delta u, \alpha \Delta^{2} u\right)=-\alpha\|\nabla \Delta u\|^{2},(\Delta u, \gamma u)=-\gamma\|\nabla u\|^{2}, \\
\left|\left(\Delta u,(\varphi(u))_{x x}\right)\right| \\
=\left|\left((\Delta u)_{x},(\varphi(u))_{x}\right)\right|=\left|\left((\Delta(u))_{x}, \varphi^{\prime}(u) u_{x}\right)\right| \\
\leq\left\|\varphi^{\prime}(u) \nabla u\right\|\|\nabla \Delta u\| \leq \frac{\alpha}{6}\|\nabla \Delta u\|^{2}+\frac{3}{2 \alpha}\left\|\varphi^{\prime}(u) \nabla u\right\|^{2},
\end{gathered}
$$




$$
\begin{aligned}
\left|\left(\Delta u,(g(u))_{y y}\right)\right| & =\left|\left((\Delta(u))_{y},(g(u))_{y}\right)\right|=\left|\left((\Delta u)_{y}, g^{\prime}(u) u_{y}\right)\right| \\
& \leq\left\|g^{\prime}(u) \nabla u\right\|\|\nabla \Delta u\| \leq \frac{\alpha}{6}\|\nabla \Delta u\|^{2}+\frac{3}{2 \alpha}\left\|g^{\prime}(u) \nabla u\right\|^{2} .
\end{aligned}
$$

According to the hypothetical condition $\left|\varphi^{\prime}(u)\right| \leq A|u|^{q},\left|g^{\prime}(u)\right| \leq B|u|^{p}$, and Sobolev interpolation inequalities

$$
\|u\|_{\infty}=C_{1}\|\nabla \Delta u\|^{\frac{1}{3}}\|u\|^{\frac{2}{3}}+C_{1}^{\prime}\|u\|,\|\nabla u\|=C_{2}\|\nabla \Delta u\|^{\frac{1}{3}}\|u\|^{\frac{2}{3}}+C_{2}^{\prime}\|u\|,
$$

so

$$
\begin{aligned}
& \frac{3}{2 \alpha}\left\|\varphi^{\prime}(u) \nabla u\right\|^{2} \leq \frac{3}{2 \alpha} A^{2} C_{1}^{2 q} C_{2}^{2}\|\nabla \Delta u\|^{\frac{2 q+2}{3}}\|u\|^{\frac{4 q+4}{3}} \leq \frac{\alpha}{6}\|\nabla \Delta u\|^{2}+C_{3}\left(C_{1}, C_{2}, q,\|u\|\right), \\
& \frac{3}{2 \alpha}\left\|g^{\prime}(u) \nabla u\right\|^{2} \leq \frac{3}{2 \alpha} B^{2} C_{1}^{2 p} C_{2}^{2}\|\nabla \Delta u\|^{\frac{2 p+2}{3}}\|u\|^{\frac{4 p+4}{3}} \leq \frac{\alpha}{6}\|\nabla \Delta u\|^{2}+C_{3}^{\prime}\left(C_{1}, C_{2}, p,\|u\|\right) .
\end{aligned}
$$

Using the Young inequality obtain

$$
(\Delta u, f) \leq\left(\nabla \Delta u,(-\Delta)^{-\frac{1}{2}} f\right) \leq \frac{\alpha}{12}\|\nabla \Delta u\|^{2}+C_{4}\left\|\Delta^{-1} f\right\|^{2} .
$$

From the (2.5) we obtain

$$
\frac{1}{2} \frac{\mathrm{d}}{\mathrm{d} t}\|\nabla u\|^{2}+\frac{\alpha}{4}\|\nabla \Delta u\|^{2} \leq-\gamma\|\nabla u\|^{2}+C_{5} .
$$

Here $C_{5} \geq C_{4}\|u\|^{2}+C_{4}^{\prime}(E)$. According to the Gronwall inequality, we can get the (2.4).

Lemma 3. Under the condition of Lemma 2, and $\left|\varphi^{\prime \prime}(u)\right| \leq A|u|^{q-1} ; \quad\left|g^{\prime \prime}(u)\right| \leq B|u|^{p-1}$; $u_{0} \in H^{2}(\Omega), f \in L^{2}(\Omega)$, so the smooth solution $u$ of Questions (1.5) - (1.7) satisfies

$$
\|\Delta u\|^{2} \leq \mathrm{e}^{-2 \gamma t}\left\|\Delta u_{0}\right\|^{2}+\frac{3\|f\|^{2}}{\gamma \alpha} .
$$

Proof. We multiply $\Delta^{2} u$ with both sides of Equation (1.5) and obtain

$$
\left(\Delta^{2} u, u_{t}+\alpha \Delta^{2} u+\gamma u+(\varphi(u))_{x x}+(g(u))_{y y}\right)=\left(\Delta^{2} u, f\right) .
$$

Here

$$
\left(\Delta^{2} u, u_{t}\right)=\frac{1}{2} \frac{\mathrm{d}}{\mathrm{d} t}\|\Delta u\|^{2},\left(\Delta^{2} u, \alpha \Delta^{2} u\right)=\alpha\left\|\Delta^{2} u\right\|^{2},\left(\Delta^{2} u, \gamma u\right)=\gamma\|\Delta u\|^{2} .
$$

By Sobolev interpolation inequality

$$
\|\nabla \Delta u\| \leq C_{0}\|\nabla u\|^{\frac{1}{3}}\left\|\Delta^{2} u\right\|^{\frac{2}{3}}+C_{0}^{\prime}\|u\| .
$$

Noticing interpolation inequalities

$$
\begin{gathered}
\|\nabla u\|_{\infty}=C_{4}\left\|\Delta^{2} u\right\|^{\frac{1}{3}}\|\nabla u\|^{\frac{2}{3}}+C_{4}^{\prime}\|u\|,\|u\|_{\infty}=C_{6}\left\|\Delta^{2} u\right\|^{\frac{1}{4}}\|u\|^{\frac{3}{4}}+C_{6}^{\prime}\|u\|, \\
\|\Delta u\|=C_{7}\left\|\Delta^{2} u\right\|^{\frac{1}{3}}\|\nabla u\|^{\frac{2}{3}}+C_{7}^{\prime}\|u\|,
\end{gathered}
$$

so 


$$
\begin{aligned}
\|\left(\Delta^{2} u,(\varphi(u))_{x x}\right) \mid & \leq\left|\left(\Delta^{2} u, \Delta \varphi(u)\right)\right| \leq\left(\left\|\varphi^{\prime}(u)\right\|_{\infty}\|\Delta u\|+\left\|\varphi^{\prime \prime}(u)\right\|_{\infty}\|\nabla u\|_{\infty}\|\nabla u\|\right)\left\|\Delta^{2} u\right\| \\
& \leq\left(A C_{6}^{q} C_{7}\left\|\Delta^{2} u\right\|^{\frac{3 q+4}{12}}\|u\|^{\frac{3 q}{4}}\|\nabla u\|^{\frac{2}{3}}+A C_{4} C_{6}^{q-1}\left\|\Delta^{2} u\right\|^{\frac{3 q+1}{12}}\|u\|^{\frac{3(q-1)}{4}}\|\nabla u\|^{\frac{5}{3}}\right)\left\|\Delta^{2} u\right\| \\
& \leq C_{8}\left(\left\|\Delta^{2} u\right\|^{\frac{39+4}{12}}+\left\|\Delta^{2} u\right\|^{\frac{3 q+1}{12}}\right)\left\|\Delta^{2} u\right\| \leq \frac{\alpha}{6}\left\|\Delta^{2} u\right\|^{2}+C_{8}^{\prime}, \\
\|\left(\Delta^{2} u,(g(u))_{y y}\right) \mid & \leq\left(\Delta^{2} u, \Delta g(u)\right) \mid \leq\left(\left\|g^{\prime}(u)\right\|\left\|_{\infty}\right\| \Delta u\|+\| g^{\prime \prime}(u)\left\|_{\infty}\right\| \nabla u\left\|_{\infty}\right\| \nabla u \|\right)\left\|\Delta^{2} u\right\| \\
& \leq\left(B C_{6}^{p} C_{7}\left\|\Delta^{2} u\right\|^{\frac{3 p+4}{12}}\|u\|^{\frac{3 p}{4}}\|\nabla u\|^{\frac{2}{3}}+B C_{4} C_{6}^{p-1}\left\|\Delta^{2} u\right\|^{\frac{3 p+1}{12}}\|u\|^{\frac{3(p-1)}{4}}\|\nabla u\|^{\frac{5}{3}}\right)\left\|\Delta^{2} u\right\| \\
& \leq C_{9}\left(\left\|\Delta^{2} u\right\|^{\frac{3 p+4}{12}}+\left\|\Delta^{2} u\right\|^{\frac{3 p+1}{12}}\right)\left\|\Delta^{2} u\right\| \leq \frac{\alpha}{6}\left\|\Delta^{2} u\right\|^{2}+C_{9}^{\prime} .
\end{aligned}
$$

According to the Young inequality,we can obtain

$$
\left(\Delta^{2} u, f\right) \leq \frac{\alpha}{12}\left\|\Delta^{2} u\right\|^{2}+\frac{3}{\alpha}\|f\|^{2} .
$$

From the (2.7) we obtain

$$
\frac{1}{2} \frac{\mathrm{d}}{\mathrm{d} t}\left\|\Delta^{2} u\right\|^{2}+\frac{7 \alpha}{12}\left\|\Delta^{2} u\right\|^{2} \leq-\gamma\|\Delta u\|^{2}+\frac{3}{\alpha}\|f\|^{2} .
$$

By the Gronwall inequality we can get the (2.6).

Lemma 4. Under the condition of Lemma 3, and

$$
\varphi(u) \in C^{3},\left|\varphi^{\prime \prime}(u)\right|+\left|\varphi^{\prime \prime \prime}(u)\right| \leq k, k>0 ; \quad g(u) \in C^{3},\left|g^{\prime \prime}(u)\right|+\left|g^{\prime \prime \prime}(u)\right| \leq l, l>0 ; \quad u_{0} \in H^{2}(\Omega), f \in H^{1}(\Omega),
$$

so the smooth solution $u$ of Questions (1.5) - (1.7) satisfies

$$
\|\nabla \Delta u\| \leq \frac{E_{0}}{t},(t>0) .
$$

Proof. We multiply $t^{2} \Delta^{3} u$ with both sides of Equation (1.5) and obtain

$$
\left(t^{2} \Delta^{3} u, u_{t}+\alpha \Delta^{2} u+\gamma u+(\varphi(u))_{x x}+(g(u))_{y y}\right)=\left(t^{2} \Delta^{3} u, f\right),
$$

Here

$$
\begin{gathered}
\left(t^{2} \Delta^{3} u, u_{t}\right)=-\frac{1}{2} \frac{\mathrm{d}}{\mathrm{d} t}\|t \nabla \Delta u\|^{2}+\left\|t^{\frac{1}{2}} \nabla \Delta u\right\|^{2}, \\
\left(t^{2} \Delta^{3} u, \alpha \Delta^{2} u\right)=-\alpha\left\|t \nabla \Delta^{2} u\right\|^{2},\left(t^{2} \Delta^{3} u, \gamma u\right)=-\gamma\|t \nabla \Delta u\|^{2} .
\end{gathered}
$$

By using the Sobolev inequality

$$
\|u\|_{\infty} \leq C_{2}\|\Delta u\|^{\frac{1}{2}}\|u\|^{\frac{1}{2}}+C_{2}^{\prime},\|\nabla u\|_{\infty} \leq C_{3}\|\Delta u\|^{\frac{3}{4}}\left\|\nabla \Delta^{2} u\right\|^{\frac{1}{4}}+C_{3}^{\prime} .
$$

So

$$
\begin{aligned}
\left|\left(t^{2} \Delta^{3} u,(\varphi(u))_{x x}\right)\right| & \leq\left|t^{2}\left(\Delta^{3} u, \Delta \varphi(u)\right)\right|=\left|t^{2}\left(\nabla\left(\varphi^{\prime}(u) \Delta u+\varphi^{\prime \prime}(u)(\nabla u)^{2}, \nabla \Delta^{2} u\right)\right)\right| \\
& \leq C\left(t^{2}\left\|\varphi^{\prime \prime}(u)\right\|_{\infty}\|\nabla u\|_{\infty}^{2}\|\nabla u\|\right)\left\|\nabla \Delta^{2} u\right\| \leq \frac{\alpha}{6}\left\|t \nabla \Delta^{2} u\right\|^{2}+C_{10},
\end{aligned}
$$




$$
\begin{aligned}
\left|\left(t^{2} \Delta^{3} u,(g(u))_{y y}\right)\right| & \leq\left|t^{2}\left(\Delta^{3} u, \Delta g(u)\right)\right|=\left|t^{2}\left(\nabla\left(g^{\prime}(u) \Delta u+g^{\prime \prime}(u)(\nabla u)^{2}, \nabla \Delta^{2} u\right)\right)\right| \\
& \leq C^{\prime}\left(t^{2}\left\|g^{\prime \prime}(u)\right\|_{\infty}\|\nabla u\|_{\infty}^{2}\|\nabla u\|\right)\left\|\nabla \Delta^{2} u\right\| \leq \frac{\alpha}{6}\left\|t \nabla \Delta^{2} u\right\|^{2}+C_{10}^{\prime} .
\end{aligned}
$$

By the Young inequality, we obtain

$$
\left|\left(t^{2} \Delta^{3} u, f\right)\right|=\left|\left(t^{2} \nabla \Delta^{2} u, \nabla f\right)\right| \leq \frac{\alpha}{6}\left\|t \nabla \Delta^{2} u\right\|^{2}+\frac{3}{2 \alpha}\|\nabla f\|^{2},
$$

From the (2.9), we obtain

$$
\frac{1}{2} \frac{\mathrm{d}}{\mathrm{d} t}\|t \nabla \Delta u\|^{2}+\frac{\alpha}{2}\left\|t \nabla \Delta^{2} u\right\|^{2} \leq C_{11}\left(\|\nabla \Delta u\|^{2}+\|\nabla f\|^{2}+1\right) .
$$

So we have

$$
\|\nabla \Delta u\| \leq \frac{E_{0}}{t},(t>0)
$$

\section{Global Attractor and Dimension Estimation}

Theorem 1. Assume that $f \in H^{1}(\Omega)$ and $u_{0} \in H^{2}(\Omega)$, so Questions (1.5) - (1.7) exist a unique smooth solution $u$ and $u \in L^{\infty}\left(0,+\infty ; H^{2}(\Omega)\right)$.

Proof. By the method of Galerkin and Lemma 1-Lemma 3, we can easily obtain the existence of solutions. Next, we prove the uniqueness of solutions in detail.

Amusse $u, v$ are two solutions of Questions (1.5) - (1.7), so the difference of them $w=u-v$ satisfies

$$
\begin{aligned}
& u_{t}+\alpha \Delta^{2} u+\gamma u+(\varphi(u))_{x x}+(g(u))_{y y}=f, \\
& v_{t}+\alpha \Delta^{2} v+\gamma v+(\varphi(v))_{x x}+(g(v))_{y y}=f,
\end{aligned}
$$

and

$$
w(0)=0, w \in L^{\infty}\left(0,+\infty ; H^{2}(\Omega)\right) .
$$

The two above formulae subtract and obtain

$$
w_{t}+\alpha \Delta^{2} w+\gamma w+(\varphi(u))_{x x}+(g(u))_{y y}-(\varphi(v))_{x x}-(g(v))_{y y}=0 .
$$

We multiply $w$ with both sides of Equation (3.1) and obtain

$$
\left(w, w_{t}+\alpha \Delta^{2} w+\gamma w+(\varphi(u))_{x x}+(g(u))_{y y}-(\varphi(v))_{x x}-(g(v))_{y y}\right)=0 .
$$

Here

$$
\left(w, w_{t}\right)=\frac{1}{2} \frac{\mathrm{d}}{\mathrm{d} t}\|w\|^{2},\left(w, \alpha \Delta^{2} w\right)=\alpha\|\Delta w\|^{2},(w, \gamma u)=\gamma\|w\|^{2} .
$$

Since the assume of Lemma 1, we obtain

$$
\begin{aligned}
\left|\left(w,(\varphi(u))_{x x}-(\varphi(v))_{x x}\right)\right| & =\left|\left(w,(\varphi(u)-\varphi(v))_{x x}\right)\right|=\left|\left(w,\left(\varphi^{\prime}(u+\theta w) w\right)_{x x}\right)\right| \\
& =\left|\left(w_{x x}, \varphi^{\prime}(u+\theta w) w\right)\right| \leq\left|\left(\Delta w, \varphi^{\prime}(u+\theta w) w\right)\right| \\
& \leq \frac{\alpha}{6}\left\|\left.\Delta w\right|^{2}+\frac{3}{2 \alpha} \varphi_{0}^{2}\right\| w \|^{2},
\end{aligned}
$$




$$
\begin{aligned}
\left|\left(w,(g(u))_{y y}-(g(v))_{y y}\right)\right| & =\left|\left(w,(g(u)-g(v))_{y y}\right)\right|=\left|\left(w,\left(g^{\prime}(u+\theta w) w\right)_{y y}\right)\right| \\
& =\left|\left(w_{y y}, g^{\prime}(u+\theta w) w\right)\right| \leq\left|\left(\Delta w, g^{\prime}(u+\theta w) w\right)\right| \\
& \leq \frac{\alpha}{6}\|\Delta w\|^{2}+\frac{3}{2 \alpha} g_{0}^{2} \|\left. w\right|^{2} .
\end{aligned}
$$

From the (3.2) we can obtain

$$
\frac{1}{2} \frac{\mathrm{d}}{\mathrm{d} t}\|w\|^{2}+\frac{2 \alpha}{3}\|\Delta w\|^{2} \leq C\|w\|^{2}, \quad C=-\gamma+\frac{3\left(\varphi_{0}^{2}+g_{0}^{2}\right)}{2 \alpha} .
$$

According to the Gronwall inequality,we obtain

$$
\|w\|^{2} \leq\|w(0)\|^{2} \mathrm{e}^{2 C t}=0 .
$$

So we can get $w=0$, the uniqueness is proved.

Theorem 2. [8] Let $E$ be a Banach space, and $\{S(t)\}(t \geq 0)$ are the semigroup operators on $E$. $S(t): E \rightarrow E, S(t) \cdot S(\tau)=S(t+\tau), S(0)=I$, here $I$ is a unit operator. Set $S(t)$ satisfy the follow conditions

1) $S(t)$ is bounded. Namely $\forall R>0,\|u\|_{\infty} \leq R$, it exists a constant $C(R)$, so that

$$
\|S(t) u\|_{E} \leq C(R)(t \in[0,+\infty)) ;
$$

2) It exists a bounded absorbing set $B_{0} \subset E$, namely $\forall B \subset E$, it exists a constant $t_{0}$, so that

$$
S(t) B \subset B_{0}\left(t>t_{0}\right) \text {; }
$$

3) When $t>0, S(t)$ is a completely continuous operator $A$.

Therefor, the semigroup operators $S(t)$ exist a compact global attractor.

Theorem 3. Under the assume of Theorem 1, Questions (1.5) - (1.7) have global attractor

$$
A=w\left(B_{0}\right)=\bigcap_{s \geq 0 t \geq s} S(t) B_{0},
$$

$B_{0}$ is the bounded absorbing set of $H^{2}(\Omega)$ and satisfies

1) $S(t) A=A, t>0$

2) $\lim _{t \rightarrow \infty} \operatorname{dist}(S(t) B, A)=0$, here $\forall B \subset H^{2}(\Omega)$ and it is a bounded set,

$$
\operatorname{dist}(X, Y)=\operatorname{supinf}_{x \in X}\|x-y\|_{H^{2}(\Omega)} .
$$

Proof. Under the conditions of Lemma 1 - Lemma 4, it exists the solution semigroup $S(t)$ of Questions (1.5)(1.7), $E=H^{2}(\Omega), S(t): H^{2}(\Omega) \rightarrow H^{2}(\Omega)$.

From Lemma 1 - Lemma 3, to $\forall B \subset H^{2}(\Omega)$ is a bounded set that includes in the ball $\left\{\|u\|_{H^{2}} \leq R\right\}$,

$$
\left\|S(t) u_{0}\right\|_{H^{2}}^{2}=\|u\|_{H^{2}}^{2} \leq\left\|u_{0}\right\|_{H^{2}}^{2}+C_{12} \leq R^{2}+C_{12}^{\prime},\left(t \geq 0, u_{0} \in B\right) .
$$

This shows $\{S(t)\}(t \geq 0)$ is uniformly bounded in $H^{2}(\Omega)$.

Furthermore, when $t \geq t_{0}=t_{0}(R,\|f\|)$, there is $\left\|S(t) u_{0}\right\|_{H^{2}}^{2}=\|u\|_{H^{2}}^{2} \leq 2\left(E_{1}+E_{2}+E_{3}\right)$, therefore,

$$
B_{0} \geq\left\{u \in H^{2}(\Omega),\|u\|_{H^{2}} \leq \sqrt{2\left(E_{1}+E_{2}+E_{3}\right)}\right\}
$$

is the bounded absorbing set of semigroup $S(t)$.

From Lemma 4, there are $\|\nabla \Delta u\| \leq \frac{E(R, t)}{t},(t>0),\left\|u_{0}\right\|_{H^{2}} \leq R$. Since $H^{3}(\Omega) \rightarrow H^{2}(\Omega)$ is tightly em- 
bedded, which means that the bounded set in $H^{3}(\Omega)$ is the tight set in $H^{2}(\Omega)$, the semigroup operator $S(t): H^{2}(\Omega) \rightarrow H^{2}(\Omega)$ to $t>0$ is completely continuous. Furthermore we can know, the attractor $\mathrm{A}$ is $w$-limited set of the absorptive set $B_{0}, \quad A=w\left(B_{0}\right)=\bigcap_{s \geq 0 t \geq s} S(t) B_{0}$.

In order to estimate the Hausdorff and fractal dimension of the global attractor A of Questions (1.5) - (1.7), let Questions (1.5) - (1.7) linearize, then we obtain

$$
\begin{aligned}
& v_{t}+\alpha \Delta^{2} v+\gamma v+\left(\varphi^{\prime}(u) v\right)_{x x}+\left(g^{\prime}(u) v\right)_{y y}=0, \\
& v_{t}+L(u(t)) v=0, \\
& v(0)=v_{0}
\end{aligned}
$$

where

$$
L(u(t)) v=\alpha \Delta^{2} v+\gamma v+\left(\varphi^{\prime}(u) v\right)_{x x}+\left(g^{\prime}(u) v\right)_{y y} .
$$

So the solutions of Questions (1.5) - (1.7) are fully smooth. It is easy to prove the initial value, appropriate, smooth and linear Questions (3.3) - (3.4) have global and smooth solutions. Let

$$
u(t)=S(t) u_{0}, v(t)=\left(D S(t) u_{0}\right) v_{0}, w(t)=S(t)\left(u_{0}+v_{0}\right),
$$

$\forall M_{1}, M_{2}$ and $T$ are constants, so it exists a constant $C_{13}=C\left(M_{1}, M_{2}, T\right)$, and $\left\|u_{0}\right\| \leq M_{1},\left\|v_{0}\right\| \leq M_{2},|t| \leq T$, so there is $\|w(t)-u(t)-v(t)\|^{2} \leq C_{13}\left\|v_{0}\right\|^{2}$.

This suggests that $S(t)$ is Frechet differential in $L^{2}(\Omega)$.

Let $V_{1}(t), V_{2}(t), \cdots, V_{N}(t)$ be the solutions of the linear Equation (3.3) corresponding to the initial value $V_{1}(0)=\xi_{1}, V_{2}(0)=\xi_{2}, \cdots, V_{N}(0)=\xi_{N}$, so there is

$$
\frac{\mathrm{d}}{\mathrm{d} t}\left\|V_{1}(t) \wedge V_{2}(t) \wedge \cdots \wedge V_{N}(t)\right\|^{2}-2 \operatorname{tr}\left(L(u(t)) \cdot Q_{N}\right)\left\|V_{1}(t) \wedge V_{2}(t) \wedge \cdots \wedge V_{N}(t)\right\|^{2}=0 .
$$

$L(u(t))=L\left(S(t) u_{0}\right)$ is linear mapping that is defined in the (3.4); $\wedge$ represents the outer product; tr represents the trace; $Q_{N}$ is the orthogonal projection from $L^{2}(\Omega)$ to the span $\left\{V_{1}(t), V_{2}(t), \cdots, V_{N}(t)\right\}$. So from (3.5), we can turn $N$ dimensions volume element $\bigwedge_{n=1}^{N} \xi_{n}$ into

$$
w_{N}(t)=\sup _{u_{0} \in A_{\xi_{n}} \in L^{2},\left|\xi_{n}\right| \leq 1} \sup _{\|}\left\|V_{1}(t) \wedge V_{2}(t) \wedge \cdots \wedge V_{N}(t)\right\|_{\Lambda_{L^{2}}^{N}}^{2} \leq \operatorname{supe}_{u_{0} \in A}\left(-\int_{0}^{t} \inf \left(t r\left(L\left(S(\tau) u_{0}\right) \cdot Q_{N}(\tau)\right) \mathrm{d} \tau\right)\right),
$$

$w_{N}(t)$ is secondly exponential, namely

$$
w_{N}\left(t+t^{\prime}\right) \leq w_{N}(t) \cdot w_{N}\left(t^{\prime}\right), t, t^{\prime} \geq 0 .
$$

So

$$
\lim _{t \rightarrow \infty} w_{N}(t)^{\frac{1}{t}}=\Pi_{n}, 1 \leq n \leq N, \Pi_{n} \leq \mathrm{e}^{-q_{N}} .
$$

Here

$$
q_{N}=\limsup _{t \rightarrow \infty}\left(\inf _{u_{0} \in A} \frac{1}{t} \int_{0}^{t} \inf \left(\operatorname{tr}\left(L\left(S(\tau) u_{0}\right) \cdot Q_{N}(\tau)\right) \mathrm{d} \tau\right)\right) .
$$

Theorem 4. Under the assume of Theorem 3, the global attractor A of Questions (1.5) - (1.7) has finite Hausdorff and fractal dimensin, and

$$
d_{H} \leq J_{0}, d_{F} \leq 2 J_{0} .
$$

Here $J_{0}$ is a minimal positive integer of the following inequality 


$$
\begin{aligned}
J_{0} \geq \frac{1}{2 \alpha C^{\prime}}\left\{6 C_{0}\left[\frac{2 C_{5}}{\gamma}\left(\left\|\varphi^{\prime \prime \prime}(u)\right\|_{\infty}+\left\|g^{\prime \prime \prime}(u)\right\|_{\infty}\right)+\sqrt{2 E_{1}+E_{2}+E_{3}}\left(\left\|\varphi^{\prime \prime}(u)\right\|_{\infty}+\left\|g^{\prime \prime}(u)\right\|_{\infty}\right)\right]\right. \\
\left.+3 C^{\prime \frac{1}{2}}\left(\left\|\varphi^{\prime}(u)\right\|_{\infty}+\left\|g^{\prime}(u)\right\|_{\infty}\right)-\gamma\right\} .
\end{aligned}
$$

Proof. By theorem [8], we need to estimate the lower bound of $\operatorname{tr}\left(L(u(t)) \cdot Q_{N}\right)$. Let $\varphi_{1}, \varphi_{2}, \cdots, \varphi_{N}$ be the orthogonal basis of subspace of $Q_{N} L^{2}(\Omega)$,

$$
\begin{aligned}
\operatorname{tr}\left(L(u(t)) \cdot Q_{N}\right) & =\sum_{j=1}^{N}\left\{\left(\alpha \Delta^{2} \varphi_{j}+\gamma \varphi_{j}+\left(\varphi^{\prime}(u) \varphi_{j}\right)_{x x}+\left(g^{\prime}(u) \varphi_{j}\right)_{y y}, \varphi_{j}\right)\right\} \\
& =\sum_{j=1}^{N}\left\{\alpha\left\|\Delta \varphi_{j}\right\|^{2}+\gamma\left\|\varphi_{j}\right\|^{2}-\left(\left(\varphi^{\prime}(u) \varphi_{j}\right)_{x},\left(\varphi_{j}\right)_{x}\right)-\left(\left(g^{\prime}(u) \varphi_{j}\right)_{y},\left(\varphi_{j}\right)_{y}\right)\right\} .
\end{aligned}
$$

Here

$$
\begin{aligned}
& \left|\left(\left(\varphi^{\prime}(u) \varphi_{j}\right)_{x},\left(\varphi_{j}\right)_{x}\right)\right| \leq\left|\left(\nabla\left(\varphi^{\prime}(u) \varphi_{j}\right), \nabla \varphi_{j}\right)\right| C \\
& =\left|\left(\varphi^{\prime \prime}(u) \nabla u \varphi_{j}, \nabla \varphi_{j}\right)+\left(\left(\varphi^{\prime}(u) \nabla \varphi_{j}\right), \nabla \varphi_{j}\right)\right| \\
& =\left|-\frac{1}{2}\left(\nabla\left(\varphi^{\prime \prime}(u) \nabla u\right), \varphi_{j}^{2}\right)+\left(\left(\varphi^{\prime}(u) \nabla \varphi_{j}\right), \nabla \varphi_{j}\right)\right| \\
& =\left|-\frac{1}{2}\left(\varphi^{\prime \prime \prime}(u)(\nabla u)^{2}, \varphi_{j}^{2}\right)-\frac{1}{2}\left(\varphi^{\prime \prime}(u) \Delta u, \varphi_{j}^{2}\right)+\left(\left(\varphi^{\prime}(u) \nabla \varphi_{j}\right), \nabla \varphi_{j}\right)\right| \\
& \leq\left\|\varphi^{\prime \prime \prime}(u)\right\|_{\infty}\|\nabla u\|_{L_{4}(\Omega)}^{2}\left\|\varphi_{j}\right\|_{L_{4}(\Omega)}^{2}+\left\|\varphi^{\prime \prime}(u)\right\|\|\Delta u\|_{\infty}\left\|\varphi_{j}\right\|_{L_{4}(\Omega)}^{2}+\left\|\varphi^{\prime}(u)\right\|\left\|\nabla \varphi_{j}\right\|^{2} \\
& \leq\left(C_{0}\left\|\varphi^{\prime \prime \prime}(u)\right\|_{\infty}\|\nabla u\|_{L_{4}(\Omega)}^{2}+C_{0}\right)+C_{0}\left\|\varphi^{\prime \prime}(u)\right\|_{\infty}\|\Delta u\|+\left\|\varphi^{\prime}(u)\right\|_{\infty}\left\|\nabla \varphi_{j}\right\|^{2} \\
& \leq C_{0} \frac{2 C_{5}}{\gamma}\left\|\varphi^{\prime \prime \prime}(u)\right\|_{\infty}+C_{0} \sqrt{2 E_{1}+E_{2}+E_{3}} \varphi^{\prime \prime}(u)\left\|\varphi^{\prime \prime}(u)\right\|_{\infty}+\left\|\varphi^{\prime}(u)\right\|_{\infty}\left\|\nabla \varphi_{j}\right\|^{2} \\
& \left|\left(\left(g^{\prime}(u) \varphi_{j}\right)_{y},\left(\varphi_{j}\right)_{y}\right)\right| \leq\left|\left(\nabla\left(g^{\prime}(u) \varphi_{j}\right), \nabla \varphi_{j}\right)\right| \\
& =\left|\left(g^{\prime \prime}(u) \nabla u \varphi_{j}, \nabla \varphi_{j}\right)+\left(\left(g^{\prime}(u) \nabla \varphi_{j}\right), \nabla \varphi_{j}\right)\right| \\
& =\left|-\frac{1}{2}\left(\nabla\left(g^{\prime \prime}(u) \nabla u\right), \varphi_{j}^{2}\right)+\left(\left(g^{\prime}(u) \nabla \varphi_{j}\right), \nabla \varphi_{j}\right)\right| \\
& =\left|-\frac{1}{2}\left(g^{\prime \prime \prime}(u)(\nabla u)^{2}, \varphi_{j}^{2}\right)-\frac{1}{2}\left(g^{\prime \prime}(u) \Delta u, \varphi_{j}^{2}\right)+\left(\left(g^{\prime}(u) \nabla \varphi_{j}\right), \nabla \varphi_{j}\right)\right| \\
& \leq\left\|g^{\prime \prime \prime}(u)\right\|_{\infty}\|\nabla u\|_{\infty}^{2}\left\|\varphi_{j}\right\|^{2}+\left\|g^{\prime \prime}(u)\right\|_{\infty}\|\Delta u\|_{\infty}\left\|\varphi_{j}\right\|^{2}+\left\|g^{\prime}(u)\right\|_{\infty}\left\|\nabla \varphi_{j}\right\|^{2} .
\end{aligned}
$$

Under the bounded condition, $\varphi_{j}(x, y)=\mathrm{e}^{i k_{1} x+i k_{2} y}$ is the standard eigenfunction of $-\Delta u=\lambda u$, and the corresponding eigenvalues are $\lambda_{j}(j=1,2, \cdots)$, and

$$
\left\|\nabla \varphi_{j}\right\|^{2}=\lambda_{j},\left\|\Delta \varphi_{j}\right\|^{2}=\lambda_{j}^{2},\left\|\varphi_{j}\right\|^{2}=1, \lambda_{j} \geq\left[\frac{(j-1)^{\frac{1}{2}}}{2}-1\right]^{2} \sim C \cdot j .
$$

Therefore, we can get 


$$
\begin{aligned}
& \operatorname{tr}\left(L(u(t)) \cdot Q_{N}\right) \geq \alpha \sum_{j=1}^{N} \lambda_{j}^{2}+N_{\gamma}-\left[C_{0} \frac{2 C_{5}}{\gamma}\left(\left\|\varphi^{\prime \prime \prime}(u)\right\|_{\infty}+\left\|g^{\prime \prime \prime}(u)\right\|_{\infty}\right)\right. \\
& +C_{0} \sqrt{2 E_{1}+E_{2}+E_{3}}\left(\left\|\varphi^{\prime \prime}(u)\right\|_{\infty}+\left\|g^{\prime \prime}(u)\right\|_{\infty}\right)+\left(\left\|\varphi^{\prime}(u)\right\|_{\infty}+\left\|g^{\prime}(u)\right\|_{\infty}\right)\left(\sum_{j=1}^{N} \lambda_{j}^{2}\right)^{\frac{1}{2}}
\end{aligned}
$$

By $\lambda_{j} \geq C_{j}^{\prime} \cdot j$ and

$$
\begin{aligned}
N>\frac{1}{2 \alpha C^{\prime}}\{ & 6 C_{0}\left[\frac{2 C_{5}}{\gamma}\left(\left\|\varphi^{\prime \prime \prime}(u)\right\|_{\infty}+\left\|g^{\prime \prime \prime}(u)\right\|_{\infty}\right)+\sqrt{2 E_{1}+E_{2}+E_{3}}\left(\left\|\varphi^{\prime \prime}(u)\right\|_{\infty}+\left\|g^{\prime \prime}(u)\right\|_{\infty}\right)\right] \\
& \left.+3 C^{\prime \frac{1}{2}}\left(\left\|\varphi^{\prime}(u)\right\|_{\infty}+\left\|g^{\prime}(u)\right\|_{\infty}\right)-\gamma\right\}=J_{0},
\end{aligned}
$$

we have

$$
\operatorname{tr}\left(L(u(t)) \cdot Q_{N}\right)>0 .
$$

Therefore

$$
d_{H} \leq J_{0}, d_{F} \leq 2 J_{0} .
$$

\section{Funding}

This work is supported by the National Natural Sciences Foundation of People’s Republic of China under Grant 11161057.

\section{References}

[1] Tian, L.X. (1994) Attractor of the Dissipated and Isolated Wave-Equation. Journal of Applied Mathmatics and Mechanics, 15, 539-547.

[2] Guo, B.L. and Li, Y.S. (1997) Attractor for Dissipative Klein-Gordon-Schrodinger Equations in $\mathrm{R}^{3}$. Journal of Differential Equations, 2, 356-377.

[3] Dai, Z.D., Guo, B.L. and Lin, G.G. (1998) Breakdown Structure of Generalized Kuramoto-Sivashinsky Equation Attractor. Applied Mathmatics and Mechanics, 19, 243-255.

[4] Du, X.Y. and Dai, Z.D. (2000) Global Attractor of Dissipative KDV Equation about Cauchy Problem. Acta Mathematica Scientia, 20, 289-295.

[5] Guo, B.L. (2000) The Infinite Dimension System. National Defense Industry Press, Beijing.

[6] Wu, J.Z., Zhao, P. and Lin, G.G. (2010) An Inertial Manifold of the Damped Boussinesq Equation. Journal of Yunnan University, 32, 310-314.

[7] Tian, L.X. and Ding, D.P. (2000) Local Property or Weakly Damped Forced KDV Equation in 2D Thin Domin. Journal of Jiangsu University of Science and Technology, 21 106-110.

[8] Lin, G.G. (2011) Nonlinear Evolution Equations. Yunnan University Press, Kunming.

[9] Wittenberg, R.W. (2012) Encyclopaedia of Mathematics. Supplement III, Kluwer, The Netherlands, 230-233.

[10] Kuramoto, Y. (1978) Diffusion-Induced Chaos in Reactions Systems. Progress of Theoretical Physics, 64, $346-367$. http://dx.doi.org/10.1143/PTPS.64.346

[11] Galaktionov, V.A., Mitidieri, E. and Pohozaev, S.I. (2009) On Global Solutions and Blow-Up for Kuramoto-Sivashinsky-Type Models, and Well-Posed Burnett Equations. Nonlinear Analysis: Theory, Methods \& Applications, 70, 2930-2952. http://dx.doi.org/10.1016/j.na.2008.12.020

[12] Makeev, M.A. and Barab A.L. (1997) Ion-Induced Effective Surface Diffusion in Ion Sputtering. Applied Physics Letters, 71, 2800-2802. http://dx.doi.org/10.1063/1.120140

[13] Bozhkov, Y. and Dimas, S. (2013) Group Classification and Conservation Laws for a Two-Dimensional Generalized Kuramoto-Sivashinsky Equation. Journal of Nonlinear Analysis, 84, 117-135. http://dx.doi.org/10.1016/j.na.2013.02.010 
Scientific Research Publishing (SCIRP) is one of the largest Open Access journal publishers. It is currently publishing more than 200 open access, online, peer-reviewed journals covering a wide range of academic disciplines. SCIRP serves the worldwide academic communities and contributes to the progress and application of science with its publication.

Other selected journals from SCIRP are listed as below. Submit your manuscript to us via either submit@scirp.org or Online Submission Portal.
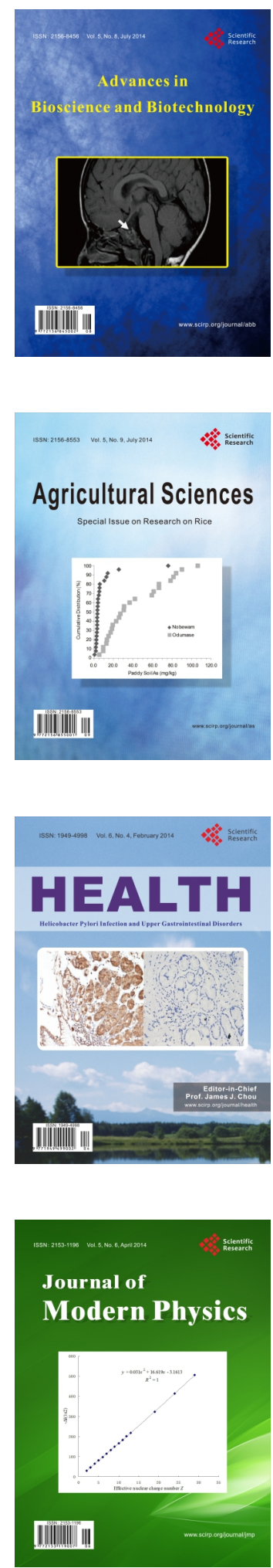
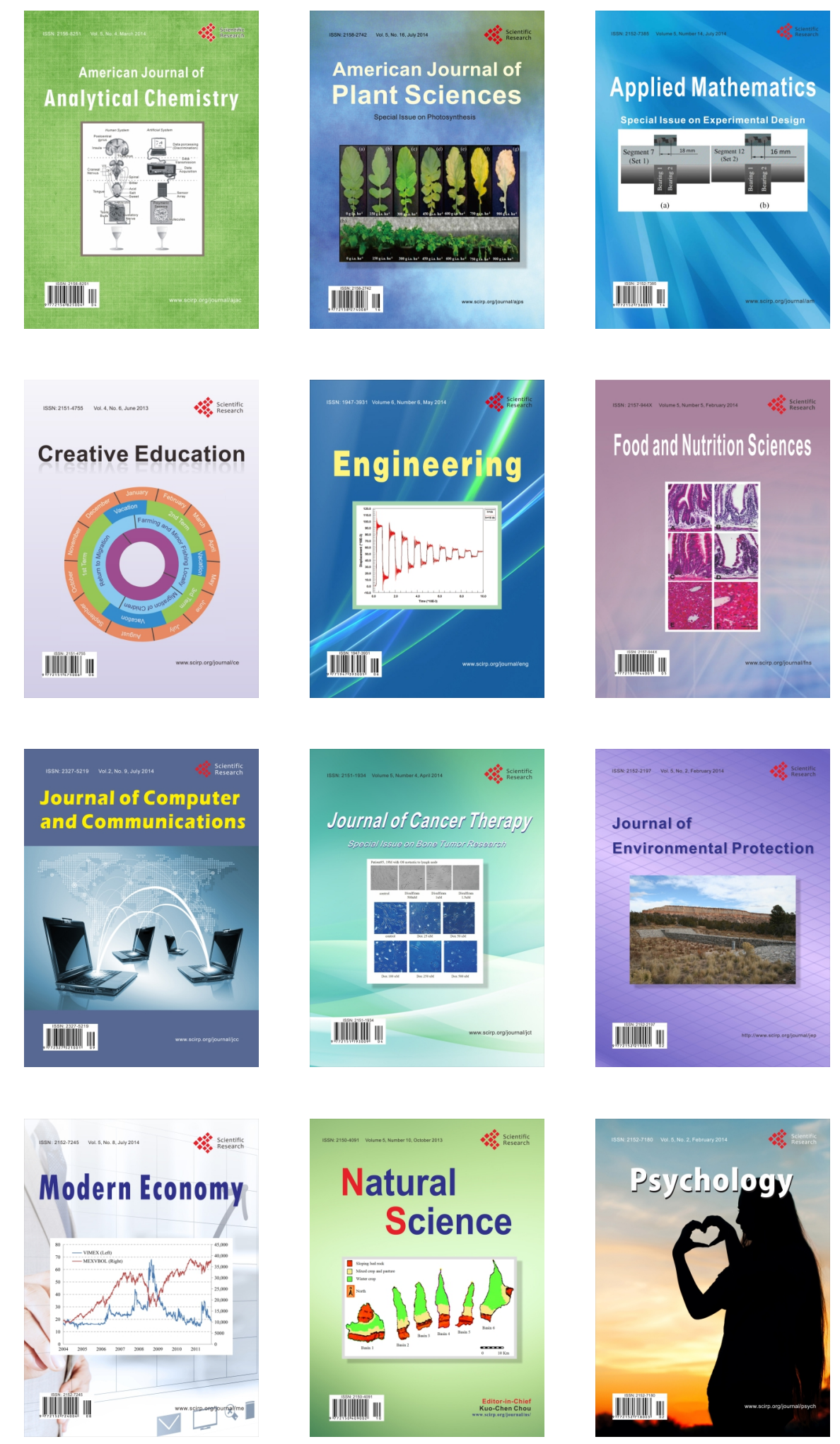